\title{
Erratum to: Reference Values of Total Lean Mass, Appendicular Lean Mass, and Fat Mass Measured with Dual-Energy X-ray Absorptiometry in a Healthy Mexican Population
}

\author{
Patricia Clark $^{1}$ - Edgar Denova-Gutiérrez ${ }^{1} \cdot$ Regina Ambrosi $^{1} \cdot$ Pawel Szulc $^{2}$. \\ Rodolfo Rivas-Ruiz ${ }^{3} \cdot$ Jorge Salmerón $^{4,5}$
}

Published online: 10 November 2016

(C) Springer Science+Business Media New York 2016

\section{Erratum to: Calcif Tissue Int (2016) 99:462-471 \\ DOI 10.1007/s00223-016-0181-z}

In the abstract and method sections the sample size should be 9523 instead of 9518 .

In the supplementary table, imprecisions between Table 1 of the above article and the supplementary tables in the sample size of some subgroups were found as well as slight differences in the estimations in some variables due to and error in rounding digits. Data have been corrected and the new version of the supplementary table is published with this erratum.

The online version of the original article can be found under doi: 10.1007/s00223-016-0181-z.

Electronic supplementary material The online version of this article (doi:10.1007/s00223-016-0207-6) contains supplementary material, which is available to authorized users.

Edgar Denova-Gutiérrez

edenovag@gmail.com

1 Unidad de Investigación en Epidemiología Clínica, Hospital Infantil de México Federico Gómez, Ciudad de México, Mexico

2 INSERM UMR, University of Lyon, Lyon, France

3 Centro Médico Nacional SIGLO XXI, Instituto Mexicano del Seguro Social, Ciudad de México, Mexico

4 Unidad de Investigación Epidemiológica y en Servicios de Salud, Instituto Mexicano del Seguro Social, Cuernavaca, Mexico

5 Centro de Investigación en Salud Poblacional, Instituto Nacional de Salud Pública, Cuernavaca, Mexico 